\title{
Texture Analysis of Deformation Induced Martensite in an AISI 301L Stainless Steel: Microtexture and Macrotexture Aspects
}

\author{
Hamilton Ferreira Gomes de Abreu ${ }^{\mathrm{a}}$, Marcelo José Gomes da Silva, \\ Luís Flávio Gaspar Herculano ${ }^{\mathrm{a}}$, Harry Bhadeshia ${ }^{\mathrm{b}}$ \\ ${ }^{a}$ Departamento de Engenharia Metalúrgica e de Materiais, Centro de Tecnologia, \\ Campus do Pici, Universidade Federal do Ceará-UFC, \\ Bloco 714, Pici, 60455-760 Fortaleza - CE, Brazil \\ ${ }^{\mathrm{b}}$ Department of Materials Science \& Metallurgy - University of Cambridge, \\ Pembroke Street, Cambridge CB2 3QZ, U.K
}

Received: November 17, 2008; Revised: June 29, 2009

\begin{abstract}
Experiments have been conducted to study the strain induced transformation from austenite to martensite in a metastable AISI 301LN austenitic stainless steel, deformed by uniaxial tension applied along rolling direction. Samples deformed 10 and 20\% have shown the presence of $\alpha^{\prime}$ martensite phase. Measured pole figures of martensite phase were compared to calculated ones, assuming no variant selection and selection of variants where interaction between stress and the plate of martensite adds to the driving force transformation variants. EBSD (electron back scatter diffraction) microtexture experiments and macrotexture X-ray diffraction were performed. The orientation distribution functions (ODFs) from measured pole figure data were calculated. The measured results were compared with calculated results in both polycrystalline and single crystal samples of austenite. The results showed that the calculated textures based in a process of variant selection consistent with Patel and Cohen's theory, which emphasizes a mechanical component of free energy, were in good agreement with measured texture.
\end{abstract}

Keywords: phase transformation, texture, martensite

\section{Introduction}

Metastable stainless steels are promising engineering materials due to a rewarding combination of good corrosion resistance and mechanical properties. Their mechanical properties can be, nevertheless, significantly affected by the deformation induced martensitic transformation for which these steels are susceptible to. Furthermore, in order to use these steels to their full potential it is important that the mechanisms acting during the martensitic phase transformation are rather completely understood.

The deformation process can induce the formation of martensite in austenitic stainless steels. The amount of martensite depends on processing parameters such as stress state of the material, temperature and rate of deformation. The steel composition and stacking fault energy can also exert great influence ${ }^{1-7}$. When shear stress is applied at temperatures near $\mathrm{M}_{\mathrm{s}}$, displacive transformation (martensitic transformation) mode is activated. When external stresses are applied the work done contributes to the free energy change; either raising or lowering the $\mathrm{M}_{\mathrm{s}}$ temperature ${ }^{8}$.

Variant selection can be understood as a phenomenon similar to the selection of slip systems among the available possibilities during single-crystal deformation ${ }^{9}$. A slip system consists of a slip plane and slip direction, for example, (111)[101] is one of 12 crystallographically equivalent systems in austenite. An applied stress is resolved on to each of the slip systems, and the ones with the highest resolved shear stress are said to be active. By analogy, the deformation due to martensitic transformation occurs on the habit plane in a displace- ment direction. When calculating the favored systems during slip deformation, the macroscopic shear on the slip planes and directions performs the selection through interaction with the applied stress. In a similar way, the interaction between the applied stress and the invariant-plane strain determines the variant selection.

The interaction energy which provides the mechanical driving force for transformation is given by ${ }^{8}$ :

$$
U=\sigma_{N} \zeta+\tau s
$$

where $\sigma_{\mathrm{N}}$ is the stress component normal to the habit plane, $\tau$ is the shear stress resolved on the habit plane in the direction of shear and $\zeta$ and s are the respective normal and shear strains associated with transformation ${ }^{10}$.

The total free energy available for transformation is the sum of chemical and mechanical components; the latter one being zero in the absence of an applied stress during transformation ${ }^{11}$ :

$$
\Delta G=\Delta G_{C H E M}+\Delta G_{M E C H}
$$

where $\Delta \mathrm{G}_{\mathrm{MECH}} \equiv U$.

It would be reasonable to assume that there is strong variant selection when the ratio of $\Delta \mathrm{G}_{\mathrm{MECH}} / \Delta \mathrm{G}$ is large ${ }^{11}$.

The transformation sequence $\gamma \rightarrow \varepsilon \rightarrow \alpha$, for austenitic stainless steels deformed by tension and rolling, has been proposed by various authors in the literature ${ }^{12-15}$. On the other hand the direct transforma- 
tion $\gamma \rightarrow \alpha^{\prime}$ through dislocation reactions has been found to be possible by $\mathrm{Nolze}^{16}$. In order to estimate the transformation texture of austenite in a stainless steel, Kundu and Bhadeshia have shown that it is not necessary to consider the two-stage transformation of austenite ${ }^{17}$. In another work ${ }^{18}$, they modelled the development of transformation texture in a deformed austenitic stainless steel using the crystallographic theory of martensite ${ }^{19,20}$ and the interaction of austenite with applied stress following Patel and Cohen's model ${ }^{8}$.

In this work, the deformation induced martensite was characterized in samples of commercial austenitic stainless steel AISI $301 \mathrm{LN}$ by a combination of XRD (X-ray diffraction) and EBSD (Electron backscattered diffraction) techniques. Texture evolution in austenite and martensite was studied by pole figures measured in single grains of the distinct phases, and also by representing the crystallographic texture with Orientation Distribution Function (ODF) of multi-grain areas of the steel. The ODF describes the frequency of occurrence of particular orientations in an Euler space ${ }^{21}$. The measured and calculated textures were compared using the crystallographic theory of martensite.

\section{Experimental}

In this study, a commercial AISI $301 \mathrm{LN}$ stainless steel bearing 17.91 Cr, 6.53 Ni, 1.80 Mn, 0.79 Si, 0.17 Mo, 0.18 Cu, 0.10 N, 0.03 C wt. (\%) and balance of Fe was used. Samples were solution treated for one hour at $1100{ }^{\circ} \mathrm{C}$. Specimens for tensile tests were fabricated in accordance with ASTM A370-3a standard, with $50 \mathrm{~mm}$ gauge length. The specimens were submitted to plastic deformations of $10,20 \%$ and up to rupture in a 25 t-Instron machine. Figure 1 shows stress $(\mathrm{MPa}) \times$ deformation $(\%)$ curves for samples deformed 10, 20\% and deformed up to rupture. Samples for pole figure measurements by XRD and EBSD were extracted from tensile test samples from the gauge length region in accordance to Figure 2.

A Philips ${ }^{\circledR}$ X'Pert Pro X-ray diffractometer with $\mathrm{Mo}-\mathrm{K}_{\alpha}$ radiation tube with an attached monochromator was used for texture studies. Three incomplete pole figures for $\alpha$ '-martensite and for austenite with maximum tilt angle of $75^{\circ}$ were measured. Orientation distribution functions (ODF) were calculated in Labotex software using ADC (arbitrary defined cells) method. Three pole figures for each phase were used in the ODF calculations. Sections of $\varphi_{2}=$ constant were plotted. The predicted texture of the product resulting from $\gamma \rightarrow \alpha$ transformation was performed with software crystal_habit_poly.f developed by Saurabh Kundu ${ }^{10}$ and available in the web address www.msm.cam.ac.uk.

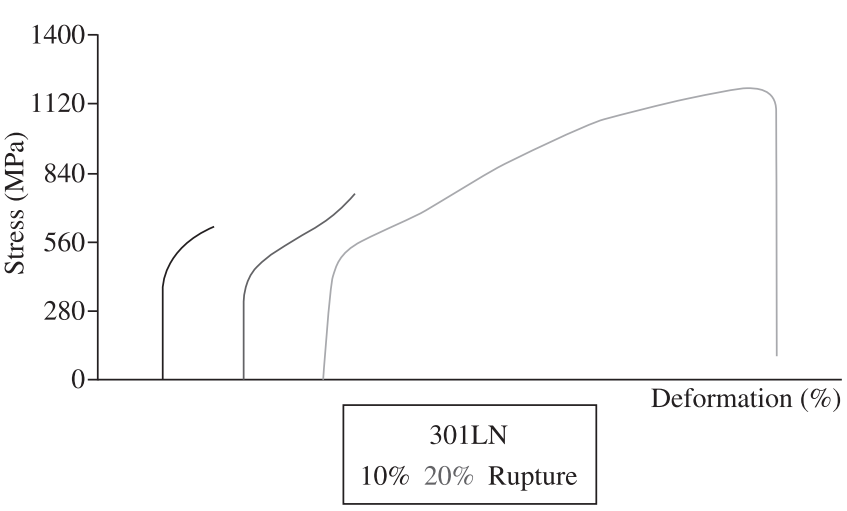

Figure 1. $\sigma \times \varepsilon$ curves for samples deformed 10,20\% and up to rupture.

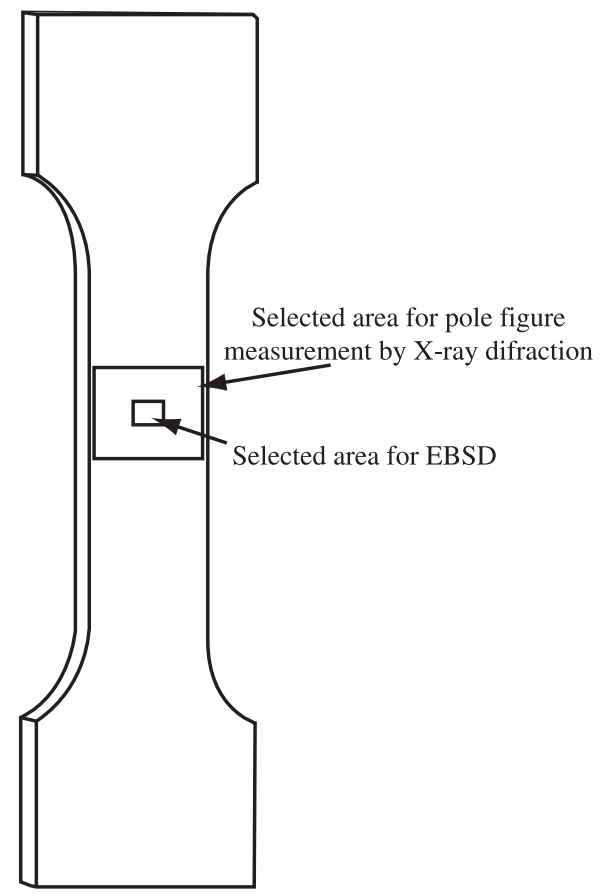

Figure 2. Sample for tensile test with indication of areas used for X-ray pole figures and EBSD analysis.

The EBSD studies were performed on samples from the mid plane section of the deformed region of the tensile specimens. An Oxford Crystal 300 EBSD system attached to a Philips ${ }^{\circledR}$ XL-30 SEM was used and the data analysed with the aid of HKL-Channel 5 software.

The same crystallographic set associated with a single martensite variant for an AISI 304 stainless steel reported by Kundu ${ }^{18}$ was used in this study. The estimated lattice parameter for austenite and martensite were 0.3589 and $0.2873 \mathrm{~nm}$ respectively. This standard set is given as follows, calculated according to reference ${ }^{9}$ :

Habit plane (-0:183989 0:596344 - 0:781359)y

Shape deformation matrix:

$$
(\gamma P \gamma)=\left(\begin{array}{ccc}
0.991342 & 0.028064 & -0.036770 \\
0.028064 & 0.909040 & 0.119180 \\
0.029429 & -0.095386 & 1.124979
\end{array}\right)
$$

Coordinate transformation matrix :

$$
(\gamma J \alpha)=\left(\begin{array}{ccc}
0.579356 & 0.542586 & 0.102537 \\
0.014470 & 0.133650 & 0.788984 \\
0.552000 & 0.572979 & 0.086936
\end{array}\right)
$$

\section{Results}

Figure 3a shows an EBSD orientation map for an un-deformed sample, whereas $3 \mathrm{~b}$ the color legend. The observed microstructure is totally $\gamma$-FCC with annealing twins inside some grains. No predominant color is observed in the map indicating absence of texture.

Figure 4 shows orientation maps for a sample after $10 \%$ of deformation for a) austenite and b) martensite. After $10 \%$ of deformation $\left(\varepsilon \sim 0.1\right.$ ), about $30 \%$ of austenite has transformed into $\alpha^{\prime}$ martensite. White regions in Figure $4 \mathrm{a}$ are $\alpha^{\prime}$ martensite. The orientation of martensite variants are in accordance with the color legend in figure $3 \mathrm{~b}$ and can be seen in fig $4 \mathrm{~b}$. The stress was applied aligned with the 


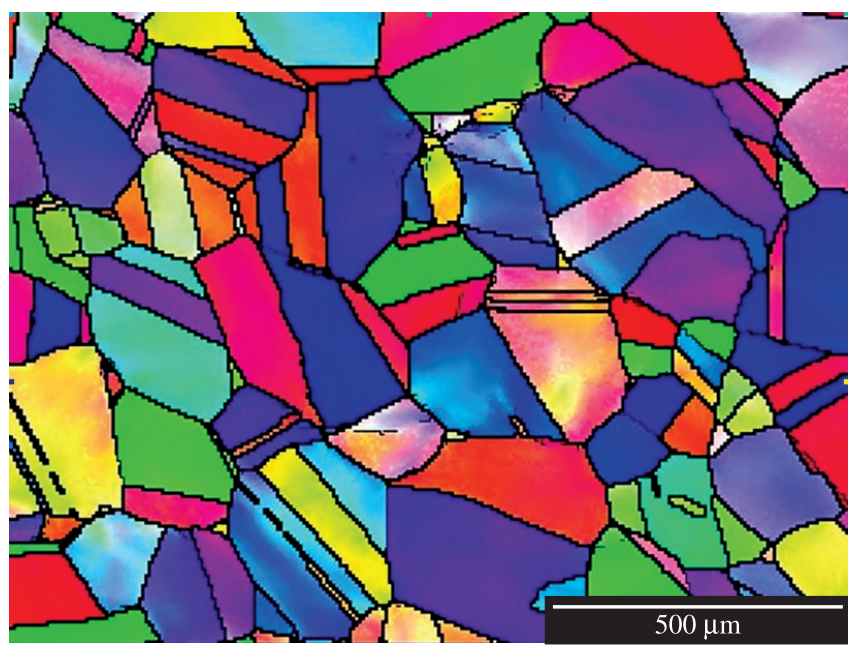

(a)

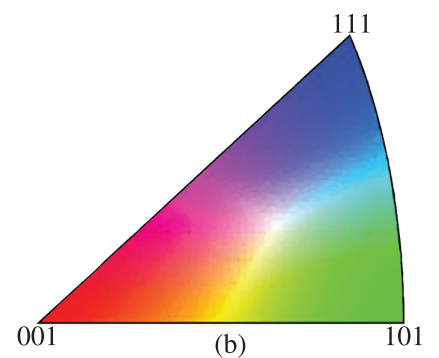

Figure 3. a) Ebsd orientation map for a sample without any deformation. b) Color legend.

vertical axis of the figure and martensite laths show a $45^{\circ}$ angle with the orientation of the applied stress. This is an indication of variant selection among martensite orientations.

Figure 5a shows the measured (100) $\alpha^{\prime}$ pole figure for the selected grain in Figure $4 \mathrm{~b}$ (lower right corner). Figures $5 \mathrm{~b}$ and $5 \mathrm{c}$ bring the calculated pole figures applying the crystallographic theory of martensite to the parent austenite grain, assuming 24 and 12 variants respectively. When comparing the measured pole figure to the one calculated for 12 variants, it is clear that there is a variant selection process and the Patel and Cohen's mode ${ }^{8}$ seems to be a very good assumption. The same procedure has been done for the $20 \%$ deformed sample $(\varepsilon \sim 0.18)$. The selected grain is the one on the right upper corner of Figure 6a. Figure 7 shows the (100) pole figures for the selected grain in Figure 6a; a) measured; b) calculated assuming no variant selection; c) calculated assuming 12 active variants. There is also a good match between measured pole figure and the calculated assuming Patel and Cohen's model ${ }^{8}$ with 12 variants acting. Some deviation can be regarded to the deformation of the BCC phase that was not taken into account in the calculation.

The software developed by Kundu based on the crystallographic theory of martensite and Patel and Cohen's model is now applied to analyze macrotexture. The austenite parent texture measured by $\mathrm{X}$-ray diffraction is presented in Figure 8. The intensity of the Copper $(\{112\}<111>)$ component increases with deformation. The intensity of the Goss component $(\{110\}<001>$ decreases with deformation. With the aid of Labotex software, 40,000 single grain orientations for

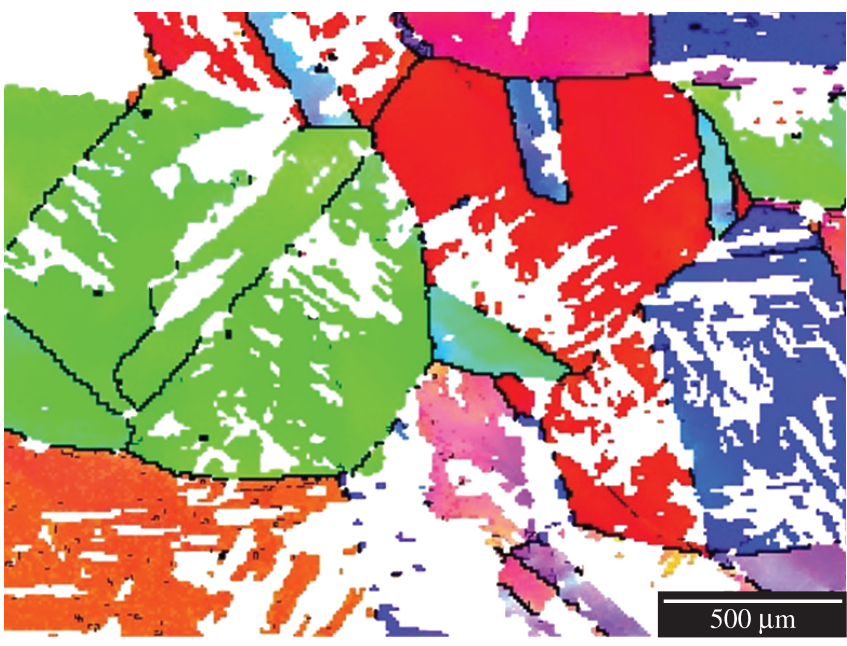

(a)

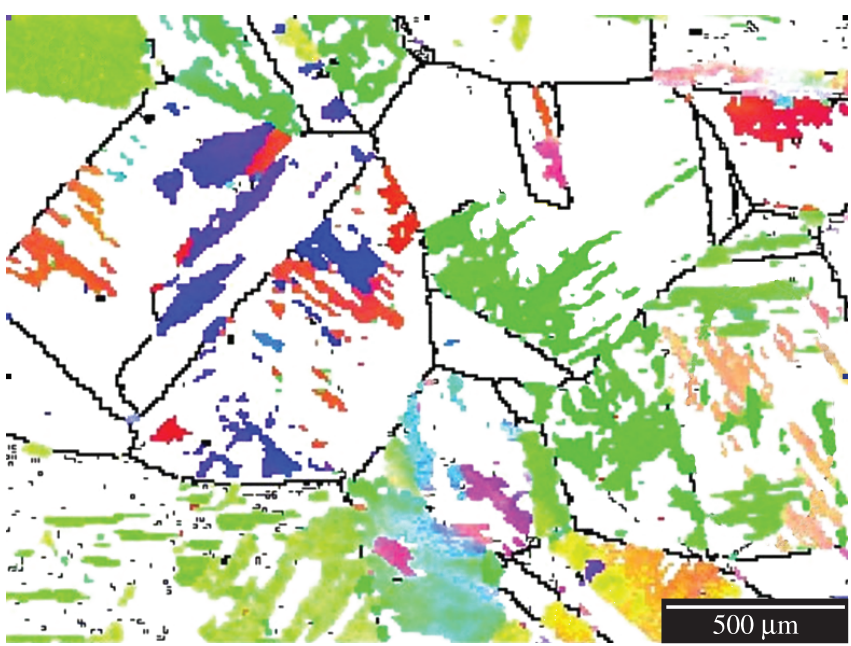

(b)

Figure 4. EBSD orientation map for a sample with $10 \%$ of deformation a) austenite b) martensite.

each texture of austenite were generated. The set of 40,000 austenite orientation matrix was used as input to the transformation texture software crystal_habit_poly. f $^{10}$. The first condition assumed was that there was no variant selection so, for each austenite single orientation 24 martensite variants were generated. The second condition was based in the Patel and Cohen's model ${ }^{8}$ and only variants with positive values of $U$ energy in equation 1 were present. It was assumed that, in average, each austenite single orientation would generate 12 variants.

$\varphi_{2}=45^{\circ} \mathrm{ODF}$ sections for the induced martensite are presented in Figures 9 and 10 for the 10 and 20\% deformed samples respectively. The first section a) of each figure shows the measured ODFs. The second section $b$ ) of each figure shows the calculated ODFs assuming no variant selection during the transformation process. Finally, the third section c) of each figure shows the calculated ODFs assuming 12 variants with higher values of energy. The shape of the measured ODFs is similar to the calculated ODFs assuming that only 12 variants were active. The calculated ODFs assuming no variant selection present for both levels of deformation show sharp texture components $\{001\}<110>$. These components were not present either in the measured ODFs or in the ODFs calculated assuming 12 active variants. Comparing the 


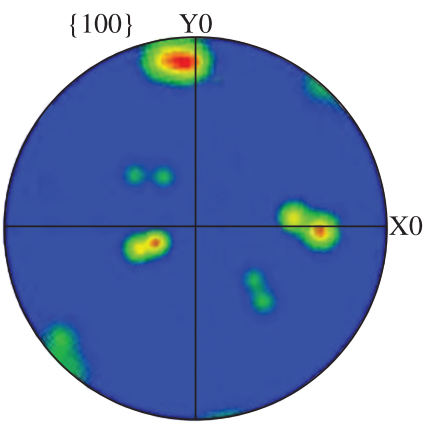

(a)

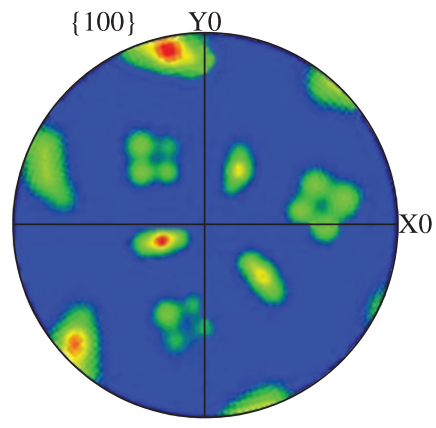

(b)

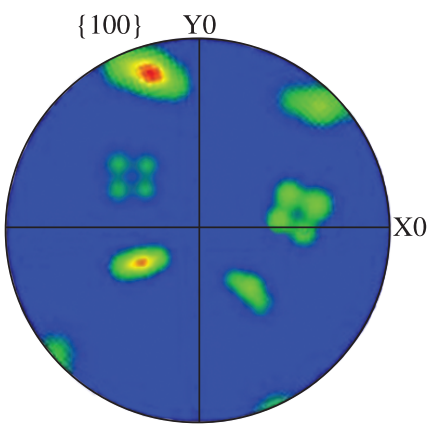

(c)

Figure 5. (100) Pole figures for the selected grain in Figure 4b; a) measured; b) calculated assuming no variant selection; c) calculated assuming 12 active variants.

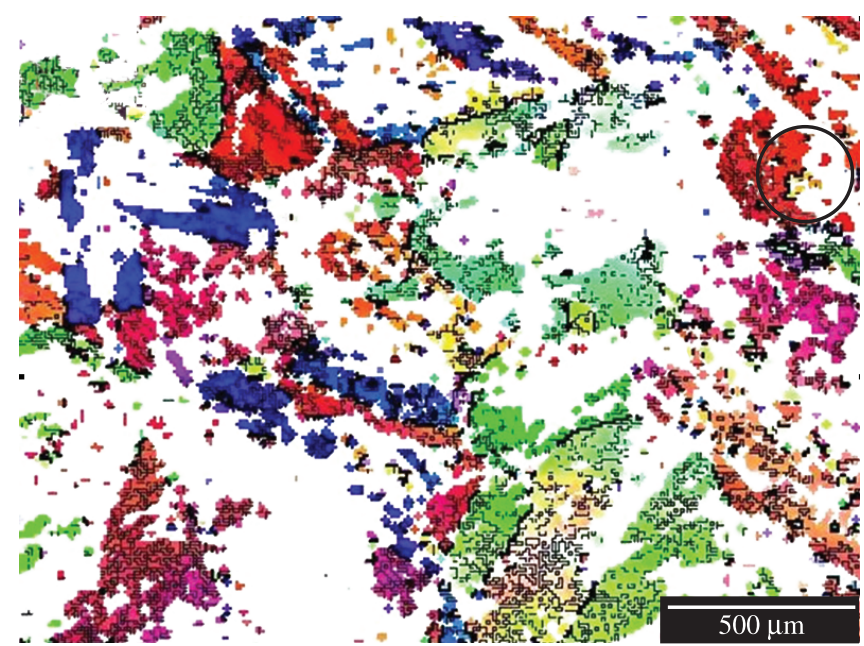

(a)

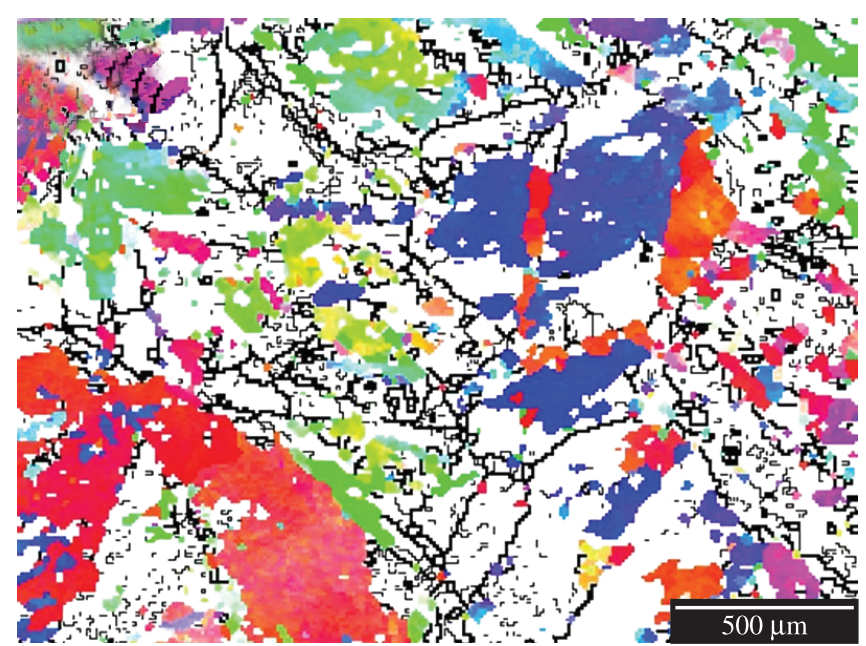

(b)

Figure 6. EBSD orientation map for a sample with $20 \%$ of deformation a) austenite b) martensite.

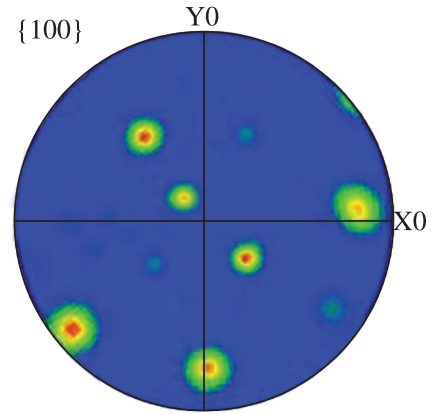

(a)

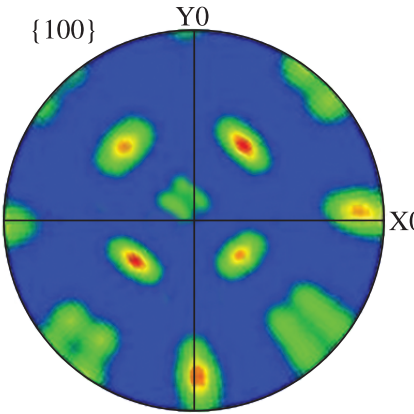

(b)

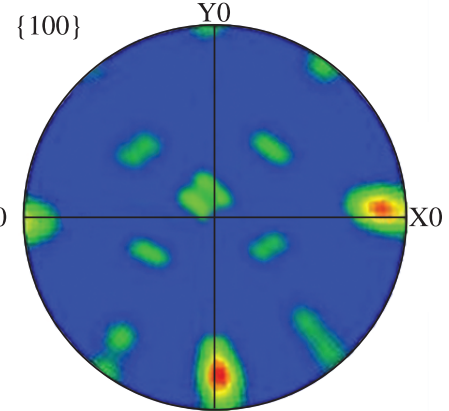

(c)

Figure 7. (100) Pole figures for the selected grain in fig. 6a; a) measured; b) calculated assuming no variant selection; c) calculated assuming 12 active variants. 


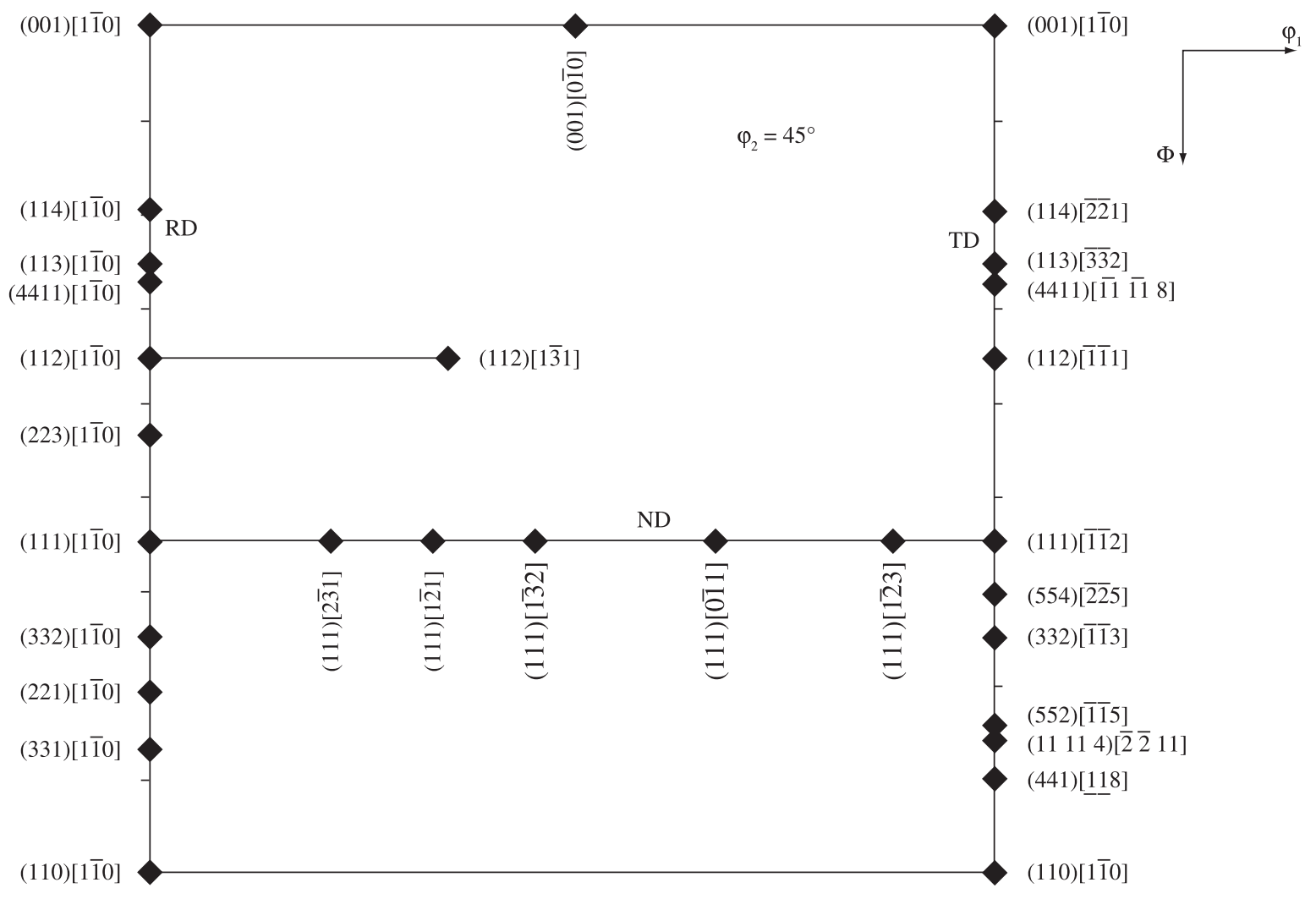

(a)

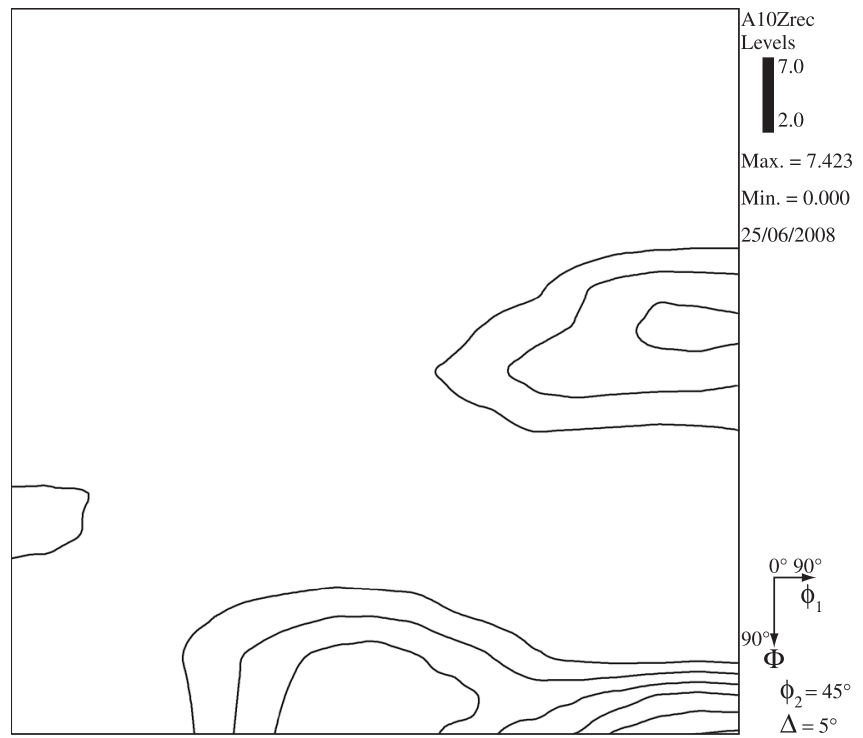

(b)

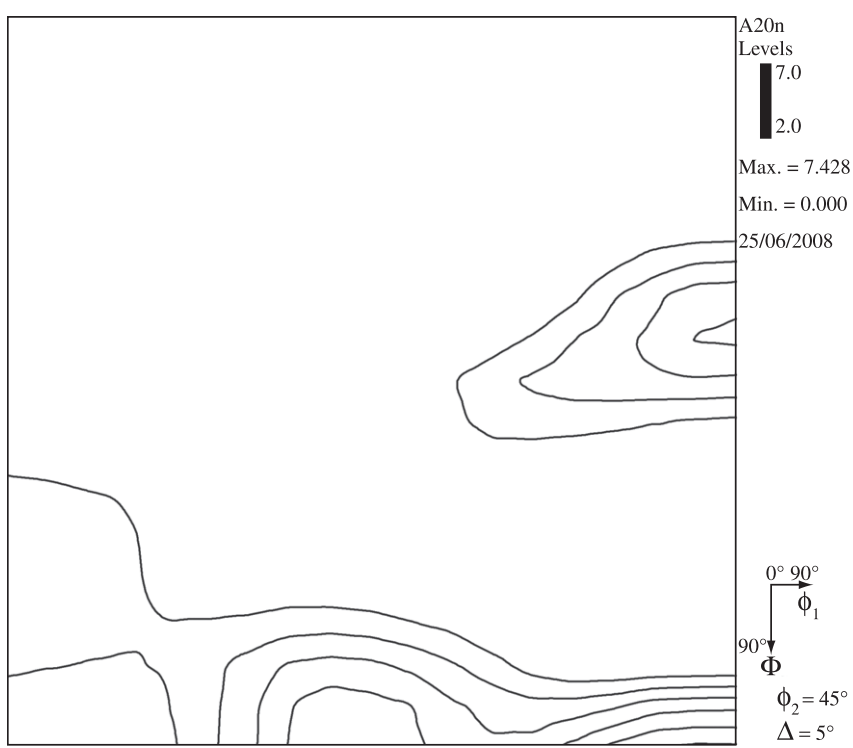

(c)

Figure 8. $\varphi_{2}=45^{\circ}$ ODF sections a) ideal orientations in the section; b) austenite deformed $10 \%$ ( $\varepsilon \sim 0.10$ ); c) austenite deformed $20 \%$ ( $\varepsilon \sim 0.18$ ). 

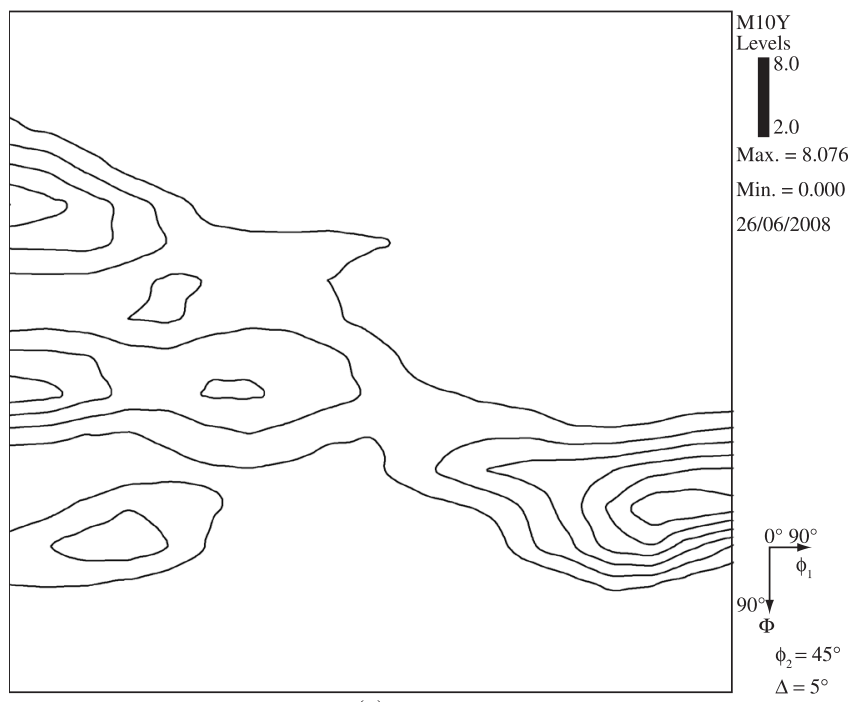

(a)

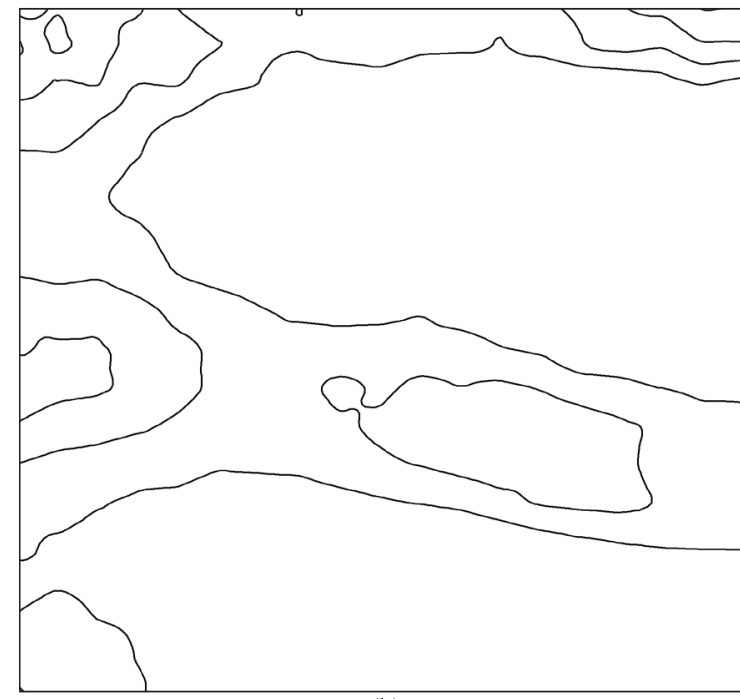

(b)

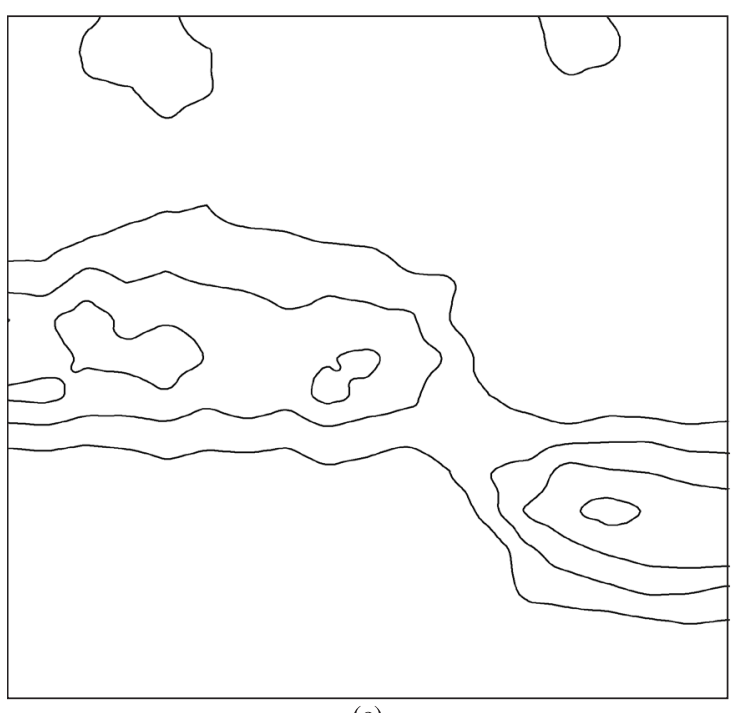

(c)

Figure 9. $\varphi_{2}=45^{\circ} \mathrm{ODF}$ sections for the induced martensite in the $10 \%$ deformed sample a) measured ODF; b) calculated ODF assuming that there is no variant selection; c) calculated ODF assuming 12 variants with higher values of energy.

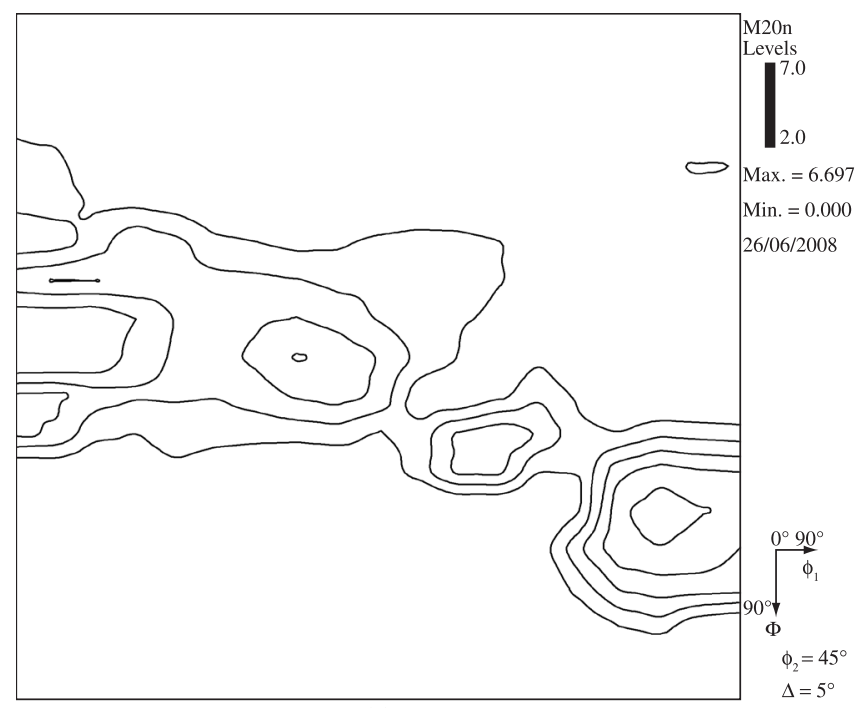

(a)

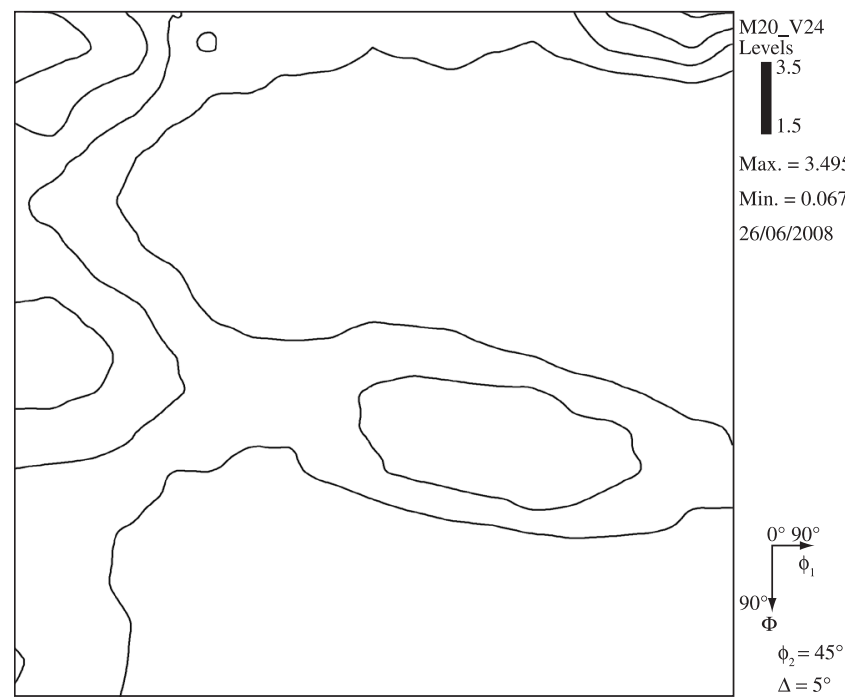

(b)

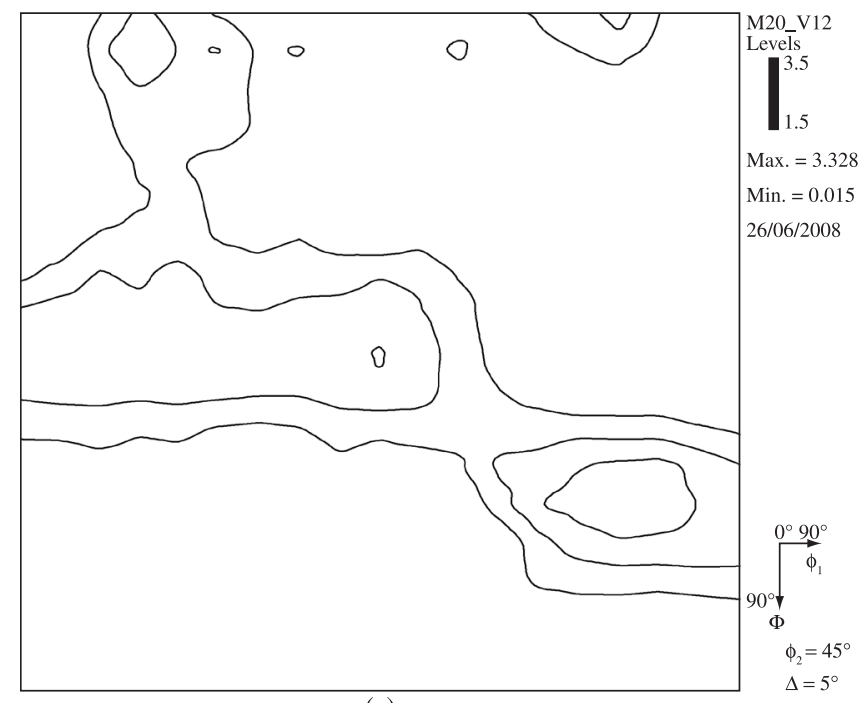

(c)

Figure 10. $\varphi_{2}=45^{\circ} \mathrm{ODF}$ sections for the induced martensite in the $20 \%$ deformed sample a) measured ODF; b) calculated ODF assuming that there is no variant selection; c) calculated ODF assuming 12 variants with higher values of energy. 
measured ODFs and the ones calculated for 12 variants it is possible to recognize that the stressed texture components were the same but the intensities on the measured ODFs were higher. One possible reason for this difference is the increase of texture with the deformation process that was not taken into account during the calculations.

\section{Conclusions}

For both 10 and $20 \%$ deformed samples, a good agreement between measured and calculated pole figures was seen when a variant selection process based on Patel and Cohen's theory, which emphasizes a mechanical component of free energy, was used. The undeformed sample presented crystallographic texture characterized by Copper $(\{112\}<111>)$ and Goss components $(\{110\}<001>)$. After deformation, the Copper component became more intense and the Goss component became less intense. A better match with the ones where the variant selection mechanism was assumed was seen. The sample with less deformation presented a better match. One possible explanation for this is the texture increase of the BCC phase with the deformation process that was not taken into account during the calculations.

\section{Acknowledgements}

We are grateful to the support from CNPq (National Council for Scientific and Technological Development), Project (200220/2007-1) and FUNCAP (Fundação Cearense de Apoio ao Desenvolvimento Científico e Tecnológico, project DCR 0053-07).

\section{References}

1. Padilha AF and Rios PR. Decomposition of Austenite in Austenitic Stainless Steels. The Iron Steel Institute of Japan-ISIJ International. 2002; 42(4): 325-337.

2. Lecroisey F and Pineau A. Martensitic Transformation in Fe-Ni-Cr-C System. Metallurgical Transactions. 1972; 3: 387-396.

3. Olson GB and M. Cohen M. Kinetics of Strain-induced Martensitic Nucleation. Metallurgical Transactions A. 1975; 6A(3): 791-795.

4. Olson GB and Cohen M. Stress-assisted isothermal martensitic transformation: Application to TRIP steels. Metallurgical Transactions A. 1982; 13A(11): 1907-1915.

5. Blanc G, Lacombe P, Baroux B and Beranger G. Stainless Steels. Paris, France: Les Editions de la Physique Les Ulis; 1993. p. 595-611.
6. Nagy E, Mertinger V, Tranta F and Sólyom J.. Deformation Induced Martensitic Transformation in Stainless Steel. Materials Science and Engineering; 2004; 378A(1-2): 308-313.

7. Choi JY and Jin W. Strain Induced Martensite Formation and its Effect on Strain Hardening Behavior in the Cold Drawn 304 Austenitic Stainless Steels. Scripta Materialia. 1997; 36(1): 99-104.

8. Patel JR and Cohen M. Criterion for the action of applied stress in the martensitic transformation. Acta Materialia. 1953; 1:531-538.

9. Bhadeshia HKDH. Geometry of Crystals. 2nd edition. [S.1.]: Institute of Materials; 2001.

10. Kundu S. Transformation Strain and Crystallographic Texture in Steels. [Ph.D. Thesis]. Cambridge: University of Cambridge; 2007.

11. Bhadeshia HKDH, David SA, Vitek JM and Reed RW. Stress-Induced Transformation to Bainite in a Fe-Cr-Mo-C Pressure Vessel Steel. Materials Science and Technology. 1991; 7:686-698.

12. Mangonon PL and Thomas G. Structure and properties of thermalmechanically treated 304 stainless steel. Metallurgical Transactions. 1970; 1(6):1577-1586.

13. Seetharaman V and Krishman P. Influence of the Martensitic Transformation on the Deformation Behavior of an AISI 316 Stainless Steel at low Temperatures. Journal of Materials Science; 1981; 16: 523.

14. Alteinberger I, Scholtes B, Martin V and Oetel H. Cyclic deformation and near surface microstructures of shot peened or deep rolled austenitic stainless steel AISI 304. Materials Science and Engineering A. 1999; 264:1-16

15. Petit B, Gey N, Cherkaoui M, Bolle B and Humpert M. Deformation Behavior and Microstructure/Texture Evolution of an Annealed 304 AISI Stainless Steel Sheet Experimental and Micromechanical Modeling. International. Journal of Plasticity. 2007; 23: 323-341.

16. Nolze G. Characterization of the fcc/bcc orientation relationship by EBSD using pole figures and variants. Zeitschrift fur Metallkunde. 2004; 95(9):744-755.

17. Kundu S and Bhadeshia HKDH. Crystallographic texture and intervening transformations. Scripta Materialia. 2007; 57: 869-872.

18. Kundu S and Bhadeshia HKDH. Transformation texture in deformed stainless steel. Scripta Materialia. 2006; 55:779-781.

19. Bowles JS and Mackenzie JK. The Crystallography of martensite transformations I. Acta Materialia 1954; 2:129-137.

20. Mackenzie JK and Bowles JS. The crystallography of martensite transformations II. Acta Materialia 1954; 2:138-147.

21. Randle V and Engler O. Introduction to texture analysis: macrotexture, microtexture and orientation mapping. Amsterdam: Gordon and Beach Science Publishers; 2000. 